\title{
LA CONQUISTA DE AMÉRICA
}

Pablo Huerga Melcón

IES Rosario de Acuña, Gijón

http://dx.doi.org/10.5209/NOMA.54286

En su libro Momentos estelares de la humanidad, Stephan Zweig solamente menciona un acontecimiento relacionado con el descubrimiento de América. Recrea allí una de las innumerables y sorprendentes gestas que llevaron a cabo los españoles, la que se refiere al descubrimiento del Océano Pacífico por parte de Vasco Núñez de Balboa. Los momentos estelares que Zweig recoge son todos ellos impresionantes, y comparten entre sí el vago conocimiento, o incluso el menosprecio por parte de los creadores de la mitología de la historia. Recuerdo, concretamente, el titulado "Un cable a través del océano", una gesta impresionante, y harto poco conocida, a pesar de su gran trascendencia histórica. En mis clases de Tecnología de la Información y la Comunicación, así como en mis clases de Comunicación audiovisual, hemos convertido este capítulo de Zweig en uno de los temas fundamentales. Y no es para menos: construir un cable e instalarlo a través del océano, para comunicar instantáneamente los dos continentes, Eurasia y América. Ciertamente, los españoles hubieron podido soñar con algo semejante hace cinco siglos. Sin embargo, llega incluso a sorprender que Zweig se haya tomado la molestia de mencionar si quiera un pequeño capítulo de la magna obra que constituye la creación del mundo hispanoamericano (sí, también del mundo moderno, pero hay que reconocer que ese mundo moderno tiene como elemento totalmente nuevo ese precisamente, el mundo hispanoamericano, y esto es algo que suele pasar desapercibido porque nadie se toma la molestia de estudiar o de entender qué es lo que ocurrió en Hispanoamérica durante todos los años que separan la llega de Colón hasta la desgraciada descomposición de Hispanoamérica, a manos de sus enemigos contumaces, fundamentalmente ingleses, franceses y holandeses). Porque no se puede negar que la historia de Hispanoamérica ha quedado enterrada sobre montañas de oprobio, sinrazón, confusión, tergiversaciones y manipulación histórica, en el contexto de los conflictos que tienen lugar entre los pueblos "desde que los hombres se alzaron de entre el barro". 
Las razones son evidentes: no existe ningún estado que la contemple como propia; ninguna de las naciones nacidas de la descomposición de Hispanoamérica pretende ese largo período. Para los españoles es solamente una mención constante que se usa para explicar los problemas internos, pero nunca se considera como propio lo que durante esos siglos está ocurriendo allí. Y las naciones hispanas de América sólo comienzan su historia propia cuando se constituyen como tal sus fronteras, esto es, en el proceso de descomposición del imperio. Así pues, nadie reivindica un período histórico de 400 años cargado de sorprendentes circunstancias. Y tengo para mí que si cada una de las naciones hispanoamericanas revisaran esa historia común, "otro gallo cantaría", como se suele decir.

Para los que nos criamos ayunos del más mínimo conocimiento sobre este evento histórico tan complejo e interesante, la historia de Vasco Núñez de Balboa está entre las que han sobrevivido al olvido y al menosprecio. Ha sobrevivido al menosprecio porque está menos involucrada, en principio, con los actos que convirtieron a los españoles en un paradigma de maldad, y porque siempre se hace necesario explicar por qué el Océano que separa América de China recibió un nombre tan irremediablemente español: Pacífico. Sorprende que, en todo caso, estas aventuras que aparecen inmaculadas, suelen quedar siempre en el haber de "Europa", o del Mundo occidental, o de Occidente, y no de España. España tendrá que cargar con otras, con las aventuras que han arrastrado valoraciones y sombras morales.

Puestos a considerar momentos estelares de la humanidad, creo que desde una escala diferente a la que utiliza Zweig, yo elegiría entre ellos, seguramente, la Atenas de Pericles, el período romano imperial inaugurado por Octavio, el descubrimiento de América, o el despliegue histórico de la revolución comunista en la Unión Soviética. De todos ellos, sin duda el más extraño, el más radical y trascendental, ha sido, creo yo, el descubrimiento y conquista de América por parte del Imperio español. Tiene en común con el primer viaje a la Luna el trascender las fronteras naturales y conceptuales sobre las que había vivido el género humano, atravesar los mares más allá de los límites de lo posible, por una parte, y por otra, encontrarse con un mundo enteramente nuevo, radicalmente aislado del resto del mundo por los siglos de los siglos. Tiene, a mi parecer, la trascendencia que podría 
tener no tanto llegar a la Luna, cuanto por ejemplo, entrar en contacto con civilizaciones extraterrestres. Así es como habría que concebir semejante encuentro, no tanto, en principio, para los españoles que con Colón pensaban llegar a Cipango, sino para los propios indios, al ver lo que se les venía encima. En la posible situación de un encuentro extraterrestre que "llegara a la Tierra", puesto que no nos es posible por ahora hacer lo contrario y colonizar otros mundos, los "terrícolas" estaríamos en la situación de los indios de América, y no al contrario, desde luego. Serían descubrimientos como el de Núñez de Balboa los que contribuirían a confirmar que se trata de un mundo enteramente nuevo, de un encuentro radicalmente inesperado. Hemos encontrado hombres en un lugar del que no hay absolutamente ninguna noticia, ningún indicio. Es cierto que otros antes iniciaron conquistas e imperios más allá de toda frontera posible. Alejandro alcanzó las montañas de Afganistán, y Adriano fortificó la isla de Gran Bretaña, pero en todo caso, esas conquistas venían precedidas de información, de indicios, de señales, de tierra firme, de conocimientos concretos y datos, de fuentes de riqueza, e incluso de un argumento para sus motivos concretos, minerales, esclavos, riquezas. Pero aquellos barcos que se propusieron atravesar el Atlántico no tenían indicios, sino sólo esperanzas, suposiciones, teorías, pero sobre todo llevaban ignorancia y miedo, también valor y arrojo en grado sumo. No es el Mediterráneo, sino el confín del mundo el que hay que sortear. Salir precisamente de Finisterre. Más que buscarlo, se encontraron con un imperio. Y su forma de construirlo es también una absoluta novedad.

El descubrimiento de América supone esencialmente incorporar al contexto de la historia universal una masa inmensa de pueblos nuevos y nuevos territorios que nunca más permanecerán fuera de ese marco universal. No sólo es importante por su cantidad, ciertamente, sino por su cualidad, y por la forma en que fueron incorporados y lo que ello supuso para el mundo. Y esa empresa es el Imperio español, nada más y nada menos. Por ello es dudoso el análisis que parte de la idea de que España perpetró una colonización como cualquiera otra de las que luego iniciaron ingleses, holandeses, franceses, alemanes o belgas. No. No es en absoluto lo mismo, porque España no permaneció impermeable a su descubrimiento, como si el plan hubiera sido buscar lo que encontraron. No. Y el resultado fue totalmente diferente porque España se prolongó en América y se convirtió en Hispanoamérica durante trescientos años. Ocurre algo parecido a lo que supone la romanización de España por parte de Roma. Roma no se puede 
concebir sin España, igual que España no se puede concebir sin América. No mantienen entre sí la relación de metrópoli a colonia, como luego será tan frecuente, porque las circunstancias que lo generan son radicalmente diferentes.

Es curioso que en España se haya dejado de considerar el tema del descubrimiento como parte de la formación de los futuros españoles. Una especie de mala conciencia colectiva se ha adveñado de nosotros de manera que procuramos evitar cualquier referencia a estos asuntos y adquieren un interés muy secundario y tangencial. ¿̇Por qué? Creo que durante muchos años hemos sufrido el triunfo de lo que se llama el pensamiento único. Según este pensamiento, es imposible conceder el más mínimo valor histórico a la empresa española en América, aunque concedemos un valor extraordinario a la llamada conquista del Oeste americano en las representaciones ideológicas que el cine de Hollywood ha hecho de este fenómeno. No importa que también aquí las armas hayan marcado la pauta, porque los objetivos son dignos. Como padre he tenido que sufrir la humillación de ver con mis hijos la película de Pocahontas, un verdadero basurero de la historia donde el malo queda asociado a España, mientras que los ingleses manifiestan un maravilloso carácter amable al conseguir la mezcla biológica de un varón inglés rubio y guapo y la más guapa de las princesas indígenas. Indigna porque eso precisamente fue lo que hicieron los españoles, y no obstante, en el caso inglés se considera algo bueno, valor positivo, mientras que en el caso de los españoles se entiende como fruto de nuestra particular depravación sexual y nuestra falta de escrúpulos. Así lo leemos, por ejemplo, en el libro de TzvetanTodorov, La conquista de América, sobre el cual vamos a hacer algunos comentarios más adelante.

La interpretación de la conquista española por parte del pensamiento único se reduce a la leyenda negra según la cual los españoles eran fundamentalmente una banda de desgraciados que ávidos de oro y riqueza entraron en América y destruyeron todo, mataron a todos los que pudieron (se habla de 70 millones), y esclavizaron a los indios y subyugaron colonialmente durante 300 años a todos los indios (que quedaron). No hubo nada positivo, ni digno de interés más allá de semejante acción. Los españoles no eran dignos de la empresa que tuvieron entre manos y todos se han encargado de demostrarlo. Esa idea no solo es muy propia del pensamiento de izquierdas en España, sino también del fundamentalismo democrático europeo y 
norteamericano, era la que yo también asumí durante muchos años hasta que decidí comenzar a estudiar el asunto un poco de manera aleatoria y desordenada, porque entre otras cosas, es difícil encontrar este asunto mínimamente estructurado y organizado, teniendo en cuenta la complejidad objetiva es explicable, pero a ello hay que añadir la insistente tendencia a ocultar para el público en general este proceso histórico. Me dejé llevar por la lectura de la Historia verdadera de la conquista de Nueva España, de Bernal Díaz del Castillo, en primer término. Impresionante lectura que deja ya al lector lleno de dudas y sorpresas. La minuciosidad de la narración que permite comprender de manera sistematica la enorme complejidad de ese acontecimiento histórico deja en suspenso aquel discurso del pensamiento único. Todo se desvanece y se complica. La calidad literaria del libro disuelve el prejuicio del analfabetismo que suele atribuirse a los conquistadores: totalmente falso. La riqueza literaria que se genera en el contexto del proceso histórico de la conquista lo desmiente absolutamente. Recuerdo, por ejemplo, el comentario de Hugh Thomas en su libro, El imperio español, donde recuerda el origen de muchas de las palabras con las que bautizaron regiones, ríos y demás accidentes geográficos los españoles, inspirados en las novelas de caballería a las que tan aficionados eran, al parecer. Cada vez se entiende mejor las razones del Quijote de Cervantes, en este contexto, evidentemente. Otras lecturas, como la de José Acosta, Historia natural y moral de las Indias, leída en medio de una visita a Potosí, me dejó sobrecogido. Hay que ser muy esforzado para alcanzar aquellas regiones, y fundar ciudades y urbanizar y organizar económicamente esos territorios, e integrarlos en una unidad estructural. La Histoira general y natural de las Indias, islas y tierra firme del mar océano, de Gonzalo Fernández de Oviedo, impresiona también por la nitidez de las descripciones, a pesar de su achacado racismo anti-indígena, según Todorov. Pero más allá de estas descripciones generales, surgen aventuras diversas, y asuntos cuya complejidad no hemos estudiado adecuadamente: la guerra civil desatada en Perú entre Pizarro y los enviados reales, las leyes de nueva planta, el debate de Salamanca entre Las Casas, Vitoria y Sepúlveda. Tampoco nos enseñaron apenas acerca del asunto de Vasco de Quiroga y su proyecto utópico con los indígenas, o la complejidad de las misiones en la actual Bolivia, o Paraguay. Las aventuras de náufragos y etnógrafos que nos permiten conocer de manera muy exhaustiva pueblos que de otro modo hubiera permanecido en el olvido, como el caso de la aventura de Cabeza de Vaca, digna de ser contada. El 
propio Bartolomé de las Casas y su propuesta política acerca de los indios que tuvo una vigencia efectiva durante todos los años que estuvo en América, como un paradigma de un modo de entender la conquista que debe ser estudiada, aunque ciertamente Las Casas es el único personaje respetado dentro de la línea del llamado pensamiento único. Asuntos sorprendentes son, por ejemplo, que a los escasos cuarenta años de conquista, ya se han construido universidades en Perú, en México, posteriormente en Córdoba, actual Argentina, y que se haya instalado ya en los años cuarenta del siglo XVI la primera imprenta en América. El trabajo de reconstrucción de las culturas precolombinas por parte fundamentalmente de los monjes que transcriben idiomas, textos y recogen información, etc., sorprende, incluso para aquellos que no estrían dispuestos a conceder nada positivo a la Iglesia Católica española en América.

Demasiada literatura, demasiados problemas, y una enorme complejidad circunstanciada que evitan que caigamos en el simplismo de la aventura de Aguirre o la cólera de Dios, con la que Herzog también quiso contribuir a echar por tierra la complejidad histórica del proceso que tuvo lugar en América. Sin embargo, el discurso dominante de la leyenda negra es una realidad inexorable que, como en otros aspectos de la historia española, solo contribuye a profundizar nuestro complejo de inferioridad. Hay que tener en cuenta que, a pesar de que hoy por hoy España es verdaderamente lo más parecido a una provincia del nuevo imperio alemán surgido de la reunificación, la nematologíade la leyenda negra sigue siendo necesaria por su contribución inestimable a la imposibilidad de construir un proyecto nacional digno en los tiempos que vivimos. La imagen de España no debe ser tampoco la que corresponde con la nación actual, porque hay que tener en cuenta que la España de hoy es un producto derivado de la propia destrucción del imperio hispanoamericano. La invasión francesa supuso el fin del imperio y su descomposición. De esa descomposición surgieron nuevas naciones, entre ellas España, México, Argentina, y otras, pero ninguna de ellas, por sí misma, existía previamente, ni siquiera España como tal. Eso es difícil de entenderlo hoy, precisamente porque miramos al pasado desde el presente geográfico y territorial actual, pero ese presente geográfico es incongruente con el pasado. El imperio español recién formado en 1492, incorporó inmediatamente los territorios conquistados que fueron reorganizados bajo un modelo común general. La España actual no es la España de entonces, sino simplemente, un trozo desgajado y 
sometido al imperio alemán. Otras naciones españolas existen en América con nombres diferentes, pero todas ellas, españolas, hispanohablantes. Creo que sería importante comenzar a entender nuestro presente histórico bajo ese prisma: somos una nación resultante del imperio español, no el imperio español reducido. La unidad que compartimos con los pueblos hispanoamericanos la da la lengua, y la historia. Por eso, cuando los franceses o los ingleses hablan del descubrimiento de América como de una empresa europea lo que están haciendo es precisamente atribuirse ellos un mérito que no tienen, y responsabilizar a España de los males que se derivaron de la conquista. Pero no es así. Inglaterra y Francia mantuvieron una incesante lucha contra el imperio español, tratando de apoderarse de sus territorios a toda costa con fines estrictamente coloniales, y su defensa no se debió a los ejércitos de la península, sino a la que realizaba cada territorio, cada virreinato dentro de su propia capacidad organizativa y militar (últimamente se ha hablado tímidamente de la gesta de Blas de Lezo, pero siempre con mucha reticencia, tampoco se conoce como debería la invasión de La Habana y otros ominosos acontecimientos que contribuyen a relativizar el juicio moral sobre el Imperio español).

Pero el discurso oficial de la leyenda negra no deja resquicio alguno para estas discusiones. Una lectura del libro de TzvetanTodorov, La conquista de América. El problema del otro, nos permite comprender el funcionamiento de este discurso. Todorov elige el caso del descubrimiento de América como un paradigma para tratar el asunto general del conflicto entre "nosotros" y "los otros", pero en cualquier caso, su juicio moral es definitivo: se trata del mayor genocidio de la historia. Los españoles son responsables del mayor genocidio, peores, en términos cuantitativos, que los nazis, o que los Jemeres Rojos. No hay nada comparable. Incluso aunque aceptemos que la gran mayoría de las víctimas indígenas se produjeron a consecuencia de las enfermedades que llevaron allí los españoles, no hay duda para Todorov. Si los españoles hubieran podido utilizarlas deliberadamente como "arma biológica", por ejemplo, lo hubieran utilizado, de modo que son responsables también de sus consecuencias. Ese es el punto de partida. Los datos de muertos en América se presentan bajo un argumento de autoridad científica indiscutible. Según él la gran mayoría de los historiadores están de acuerdo en atribuir a España la muerte de 70 millones de indígenas. No es necesario citar fuentes, o presentar argumentos y datos, baste decirlo así. No dedica mucho tiempo, no es 
necesario, nadie lo va a discutir, simplemente ocurrió. Una vez puestas así las cosas, la verdad es que resulta bastante extraño que siga el libro analizando datos. La pregunta es por qué.

El libro estudia el caso de la conquista española durante los cien primeros años, de los casi cuatrocientos que ocupa la conquista desde 1492 hasta 1898. Y de esos cien primeros años, sólo estudia lo que se refiere a México y el Caribe. Es suficiente. Los juicios de valor se suceden sutilmente a lo largo del libro. Todorov va asignando a cada uno el nivel de responsabilidad sobre lo ocurrido. Colón, ciertamente, está exento de males. Su interés es extender la fe y recrear el viaje de Marco Polo, como no sabe a dónde ha llegado, se limita a convencer a los españoles para que le sigan. Los españoles, como no podía ser de otra manera, sólo se convencen con el oro, y él entonces utiliza ese argumento, pero sus objetivos son otros, solamente pretende cristianizar. Cortés, español, ya da muestras de toda la perfidia. Si promueve los matrimonios mixtos de sus soldados es solamente con el fin de someterlos, si promueve el estudio de su lengua, es solamente para conocer mejor sus intenciones y poder someterlos, si fomenta la enseñanza del español, es para dominarlos y aculturizarlos, en cualquier caso, es culpable de no respetar a los indígenas y sus costumbres.

Las Casas es el más interesante: sólo queda exculpado al final de su vida, cuando sus escritos inducen a pensar en un deseo no explícito de que esas tierras se independicen de España, mientras tanto y hasta que llega a esas conclusiones, sigue siendo un español envilecido y no entiende a los indios. El mayor peligro del discurso más generalizado de Las Casas es precisamente haber defendido su igualdad, ese fue su pecado y la razón de que deba ser condenado: "El postulado de igualdad lleva consigo la afirmación de identidad, y la segunda gran figura de la alteridad, aun si es indiscutiblemente amable, nos lleva hacia un conocimiento del otro todavía menor que la primera" (pág. 181). Es decir, Las Casas, al defender su igualdad está precisamente faltando el respeto al otro, al no considerar su diferencia, por tanto, sigue preso en la misma actitud reprochable: "hemos visto que, precisamente porque era cristiano, percibía mal a los indios." Y se pregunta profundamente Todorov: "¿Puede uno querer realmente a alguien si ignora su identidad, si ve, en lugar de esa identidad, una proyección de sí o de su ideal?" Francamente, Las Casas y todo su amor, han sido derribados. Ciertamente, Las Casas era cristiano, y por más defensor de los indios que era, lo era precisamente en tanto que 
cristiano, de modo que este producto dialéctico que nace en la polémica española acerca de los indios, que tantas veces se ha utilizado como paradigma de justicia, etc., no deja de formar parte de esa forma española de imponerse al otro. "Ahora bien, -dice Todorov-, ¿no hay ya una violencia en la convicción de que uno mismo posee la verdad, cuando ése no es el caso de los otros, y que, además, hay que imponerla a esos otros?" (pág. 182). Nuevamente, tenemos que asentir. No hay perdón para el genocidio que adquiere sus formas más perversas en estas falsas figuras de justicia que sólo contribuyeron a difuminar la imagen del mayor holocausto de la historia. Todorov, sin embargo, perdona a Las Casas: "el reconocer que la ideología asumida por Las Casas y otros defensores de los indios es efectivamente una ideología colonialista no mengua en nada la grandes del personaje, sino al contrario. Justamente porque no podemos dejar de admirar al hombre, importa juzgar con lucidez su política". (pág. 186).

No obstante, Las Casas quedará redimido, según Todorov, en virtud del cambio de perspectiva que obra en él a partir del año 1550, cuando Las Casas comienza a comprender el verdadero valor de los sacrificios humanos de los aztecas: "En una primera etapa, Las Casas afirma que, incluso si el canibalismo y el sacrificio humano son condenables en sí, no se sigue de ello que haya que declarar la guerra a quienes lo practican: el remedio tiene entonces el riesgo de ser peor que la enfermedad. A eso se añde el respeto, que Las Casas supone común a indios y españoles, por las leyes del país. Si la ley impone el sacrificio, al practicarlo uno se conduce como un buen ciudadano, y no se puede culpar al individuo por hacerlo. Pero después da un paso más: la condena misma se vuelve problemática." (pág. 196). De hecho, también el cristianismo incluye el sacrificio humano. Y por fin, reconce Las Casas que no habiendo cosa alguna más importante para los hombres que su vida, "deben inmolar incluso víctimas humanas al Dios veradero o al falso, si es tenido por verdadero, para que, al ofrecerle así la cosa más preciosa, se muestren especialmente agradecidos por tantos beneficios recibidos" (pág. 200).

Todorov aplaude estos argumentos que considera propios del "perspectivismo" (en vez de relativismo), y al menos reconoce y se asombra de que siendo España para él cosa tan ominosa hayan dejado vivo y coleando a este sujeto hasta el final de sus días sin quemarle a fuego vivo como hicieron en Roma con G. Bruno en 1600, sobre todo cuando alcanza su propuesta política final, que es la de 
sugerir "al rey de España, ni más ni menos, que renuncie a sus posesiones allende el Atlántico. Y la única guerra que considera sería la que hiciera el rey contra los conquistadores españoles (pues Las Casas sospecha que éstos no querrán renunciar de buen grado a sus conquistas)" (p. 204). Las Casas renuncia por fin "al deseo de asimilar a los indios, y elige la vía neutral: los indios mismos decidirán sobre su propio porvenir." (pág. 204).

Todorov no lo menciona, pero hubo muchos e importantes casos en la inmediata historia de Hispanoamérica en donde los requerimientos de los reyes hubieron de ser impuestos a los conquistadores mediante auténticas guerras civiles, como en el caso de Bolivia y Perú con Pizarro, por ejemplo. De manera que Las Casas no alude a una guerra de salvación ideal del rey a favor de los indios, sino de efectivos casos de resistencia a renunciar a prácticas prohibidas por la corona desde el principio y de manera insistente. Pero en el Holocausto más grande de la historia cualquier intento de análisis comprensivo es inútil.

Recoge también Todorov las ordenanzas definitivas de Felipe II de 1573, en las que las tesis de Las Casas hacen furor, pero reconoce que al final, desterrar la esclavitud, conservar los jefes locales, evitar la conversión forzada, la pacificación a ultranza, todo ello no significa más que suavizar con palabras lo que sigue siendo, de hecho, conquista y latrocinio. Según Todorov estas leyes sólo sirven para generar una imagen positiva: "El texto no podría ser más explícito a este respecto: lo que hay que desterrar no son las conquistas, sino la palabra "conquista"; la "pacificación" no es sino otra palabra para designar lo mismo, pero no creamos que es vana esa preocupación lingüística. Luego, hay que actuar al amparo del comercio, manifestando amor, sin mostrar codicia." AL fin y al cabo, "el objetivo global [del Requerimiento] no se modifica: sigue siendo la sumisión de esas tierras a la corona de España." (pág. 188). Todorov no especifica en qué consiste esa sumisión, cómo se reglamenta y se estructura, eso no tiene ningún interés, porque ya en principio, es todo reprobable y perverso. Por tanto, esas leyes que manifiestan un planteamiento tan distante al de otros imperios y procedimientos no engaña a Todorov. Incluso cuando esas leyes promueven el desarrollo y la intensificación de la educación para los indios (recordemos la fundación de universidades por toda Hispanoamérica ya desde los primeros años de la conquista), sólo sirven para lo mismo: "En cuanto a la enseñanza, ofrecida generosamente a los hijos de los nobles, no es más que un pretexto para 
apoderarse de ellos y utilizarlos, si se da el caso, como medio de coerción (vuestros hijos en nuestras escuelas son rehenes... [sic])"

Amables juicios emite Todorov en defensa de los indígenas contra los españoles. La perversión de España no tiene límites. Si puso en marcha la etnografía, lo hizo sólo con fines imperialistas: "sabemos que España es el primer país colonial que aplica sistemáticamente este precepto, gracias a las investigaciones emprendidas por instigación de la corona [nótese que dice "instigación", y no por ejemplo, "fomento", porque Todorov ha enjuiciado y debe seguir manteniendo el tono]." (pag. 188). Es más, la cosa alcanza unos grados de perversión intolerables, porque cuando los religiosos cristianos promueven el cuidado del cuerpo y la salud atendiendo a los indígenas, esto será no para que estén sanos, sino para dominarlos mejor. Cuanto más sanos estén, mejor trabajarán y eso redunda siempre en beneficio de España: "el sujeto será tanto más productivo cuanto mejor cuidado esté. Los religiosos proporcionarán entonces cuidados médicos por una parte, instrucción por otra [...] La salud del cuerpo y la del alma estarán en lo subsiguiente al cuidado de especialistas laicos: el médico y el profesor." (189).

Todorov se refiere ahora a Francisco de Vitoria, el defensor de la idea de la guerra justa, que legitimará por ejemplo la conquista de Cortés, en el contexto del conflicto que existía entre Txlascaltecas y Aztecas. Aquí Todorov condena explícito: "Vitoria habla como si la guerra entre mexicanos y tlaxcaltecas fuera una relación básica, donde los españoles sólo intervendrían en su calidad de aliados de estos últimos. Pero sabemos que eso es una brutal [sic] transformación de la realidad; aquí Vitoria es culpable [sic] de haberse fiado de la aproximación de los "se dice", de los decires de "los que han estado alli" [...] sin que haya realmente "búsqueda de la verdad"." (193). Vitoria es culpable. Triste cosa, nuestro Vitoria, encumbrado por Obama en su discurso del Premio Nobel de la Paz, culpable. ¿Hay guerras justas e injustas dependiendo de la "verdad en sí"? ¿̀No decide la justicia de la guerra precisamente el que la gana? En aquel tiempo, había ganado España, pero hoy, como demuestra Todorov, España ha sido derrotada. Creo que, en cualquier caso, el lector debería echar un vistazo a la relación que del asunto hace Bernal Díaz del Castillo. La relación deja pocas dudas al respecto de cuál fue el proceder del grupo de españoles de Cortés cuando se encontraron con los Tlascaltecas. 